\title{
Characterization of GPR101 transcript structure and expression patterns
}

\author{
Giampaolo Trivellin', Ivana Bjelobaba², Adrian F Daly3, Darwin O Larco4, \\ Leonor Palmeira3, Fabio R Faucz', Albert Thiry5, Letícia F Leal1,6, Liliya Rostomyan³, \\ Martha Quezado7, Marie Helene Schernthaner-Reiter1, Marija M Janjic², \\ Chiara Villa3,8, T John Wu4, Stanko S Stojilkovic², Albert Beckers3, \\ Benjamin Feldman ${ }^{9}$ and Constantine A Stratakis ${ }^{1}$
}

\author{
1Section on Endocrinology and Genetics, Eunice Kennedy Shriver National Institute of Child Health and \\ Human Development (NICHD), National Institutes of Health (NIH), Bethesda, Maryland, USA \\ 2Section on Cellular Signaling, Eunice Kennedy Shriver National Institute of Child Health and Human \\ Development (NICHD), National Institutes of Health (NIH), Bethesda, Maryland, USA \\ 3Department of Endocrinology, University of Liège, Domaine Universitaire du Sart-Tilman, Liège, Belgium \\ ${ }^{4}$ Department of Obstetrics and Gynecology, Uniformed Services University of the Health Sciences, \\ Bethesda, Maryland, USA \\ ${ }^{5}$ Department of Pathology, University of Liège, Domaine Universitaire du Sart-Tilman, Liège, Belgium \\ ${ }^{6}$ Department of Pediatrics, University of Sao Paulo, Ribeirao Preto, São Paulo, Brazil \\ 7Laboratory of Pathology, National Cancer Institute (NCI), National Institutes of Health (NIH), Bethesda, \\ Maryland, USA \\ ${ }^{8}$ Hopital Foch, Service d'Anatomie et Cytologie Pathologiques, Suresnes Cedex, France \\ ${ }^{9}$ Division of Developmental Biology, Eunice Kennedy Shriver National Institute of Child Health and \\ Human Development (NICHD), National Institutes of Health (NIH), Bethesda, Maryland, USA
}

Correspondence should be addressed to C A Stratakis Email stratakc@mail.nih.gov

\begin{abstract}
We recently showed that Xq26.3 microduplications cause X-linked acrogigantism (X-LAG). X-LAG patients mainly present with growth hormone and prolactin-secreting adenomas and share a minimal duplicated region containing at least four genes. GPR101 was the only gene highly expressed in their pituitary lesions, but little is known about its expression patterns. In this work, GPR101 transcripts were characterized in human tissues by 5'-Rapid Amplification of CDNA Ends (RACE) and RNAseq, while the putative promoter was bioinformatically predicted. We investigated GPR101 mRNA and protein expression by RT-quantitative PCR (qPCR), whole-mount in situ hybridization, and immunostaining, in human, rhesus monkey, rat and zebrafish. We identified four GPR101 isoforms characterized by different 5'-untranslated regions (UTRs) and a common $6.1 \mathrm{~kb}$ long 3'UTR. GPR101 expression was very low or absent in almost all adult human tissues examined, except for specific brain regions. Strong GPR101 staining was observed in human fetal pituitary and during adolescence, whereas very weak/absent expression was detected during childhood and adult life. In contrast to humans, adult monkey and rat pituitaries expressed GPR101, but in different cell types. Gpr101 is expressed in the brain and pituitary during rat and zebrafish development; in rat pituitary, Gpr101 is expressed only after birth and shows sexual dimorphism. This study shows that different GPR101 transcripts exist and that the brain is the major site of GPR101 expression across different species, although divergent species- and temporal-specific expression patterns are evident. These findings suggest an important role for GPR101 in brain and pituitary development and likely reflect the very different growth, development and maturation patterns among species.
\end{abstract}
Key Words
- GPR101
- pituitary
- hypothalamus
- expression analysis
- transcripts structure

Journal of Molecular

Endocrinology

(2016) 57, 97-111 


\section{Introduction}

$\mathrm{X}$-linked acrogigantism (X-LAG) is a recently discovered syndrome characterized by early childhood-onset gigantism due to Xq26.3 microduplications (Trivellin et al. 2014). We have shown that microduplications in $\mathrm{X}$-LAG patients can occur constitutionally or as somatic mosaicism (Daly et al. 2016b) as was recently confirmed elsewhere (Iacovazzo et al. 2016, Rodd et al. 2016). Patients with X-LAG present with mixed growth hormone (GH) and prolactin (PRL)-secreting pituitary macroadenomas and/or hyperplasia before 5 years of age (Beckers et al. 2015). We have previously shown that the smallest common duplicated region shared by all patients is about $500 \mathrm{~kb}$ long and contains four OMIM genes, of which only an orphan G protein-coupled receptor (GPCR) named GPR101 (OMIM\# 300393) is highly expressed in pituitary tumor tissue. Functional studies showed that GPR101 duplication is the likely cause of X-LAG (Trivellin et al. 2014). The very recent finding of a patient harbouring a duplication of only the GPR101 gene supports our determination of its causative role in X-LAG (Iacovazzo et al. 2016).

GPR101 belongs to the class A rhodopsin superfamily of GPCRs (Kakarala \& Jamil 2014). Phylogenetic analysis showed that it is most similar to another orphan GPCR named GPR161 (Mukhopadhyay et al. 2013). Both GPCRs are closely related to the adrenergic and serotonin receptors, but share only about 30\% sequence identity in their transmembrane regions (Lee et al. 2001). So far, very little is known regarding which tissues express GPR101, especially in humans. We and others have previously shown that Gpr101 is highly expressed in the hypothalamus in rodents, where it seems to play a role in hypothalamic control of energy homeostasis (Lee et al. 2001, Bates et al. 2006, Nilaweera et al. 2007, 2008, Regard et al. 2008, Trivellin et al. 2014). Interestingly, an increase in Gpr101 mRNA expression in the rat hypothalamus was seen after puberty and from late pregnancy to late lactation suggesting that the receptor may also play a role in physiological processes associated with pregnancy, lactation and reproduction (Nilaweera et al. 2008) (E Beadle, D O Larco, A Vana, M Cho-Clark and T J Wu, personal communication). On the contrary, few cells seem to express GPR101 in the adult human hypothalamus. Normal pituitary and GH-secreting tumors without the Xq26.3 duplication also show relatively scarce or negligible GPR101 expression (Trivellin et al. 2014).

The complete structure of the human GPR101 gene is unknown as only the coding sequence (CDS) has been characterized to date (NM_054021.1/ ENST00000298110.1). The putative GPR101 transcript is 1527 base pairs (bp) long and consists of one proteincoding exon. The structure of the Gpr101 gene has been better characterized in mouse (NM_001033360.3) and rat (NM_001108258.1) and in both species it consists of two exons, of which only exon 2 is protein-coding. In zebrafish, a gpr101 ortholog has been predicted and annotated (ENSDARG00000039218). The human GPR101 gene shares 80,79 and 55\% similarity with the mouse, rat and zebrafish orthologs, respectively (http://www.ncbi. nlm.nih.gov/homologene/).

We undertook this study to better characterize GPR101 expression across different species, and to define the structure of the human gene.

\section{Materials and methods}

\section{Animal care and use}

All animal experiments were approved by the National Institute of Child Health and Human Development (NICHD) Animal Care and Use Committee (ACUC).

\section{In silico analyses}

Alignment of the mouse Gpr101 mRNA (NM_001033360.3) to the human genome (GRCh37/h19 assembly) and of the human putative GPR101 promoter region to the mouse genome (GRCm38/mm10) was performed with the BLAST tool (http://blast.ncbi.nlm.nih.gov/Blast.cgi) using the discontiguous megablast algorithm. Analysis of the human putative GPR101 promoter region was also performed with other three software packages: MPromDb (http:// mpromdb.wistar.upenn.edu/search), the University of California, Santa Cruz (UCSC) genome browser track annotating transcription factors (TFs) binding sites assayed by ChIP-seq (TF ChIP-seq (161 factors) from ENCODE with Factorbook Motifs), and the MatInspector tool from the Genomatix Software Suite (http://www.genomatix.de/). Genomatix results were filtered by vertebrate elements, location (only sites located within or spanning the conserved human-mouse regions were considered), a matrix similarity threshold of 0.85 , and strand (only for TATA box elements, since true positives are considered those in the same orientation of the gene).

\section{RNAseq and rapid amplification of cDNA ends}

Total RNA was extracted from two female patients with X-LAG (both from lymphocytes and pituitary tumors)

Published by Bioscientifica Ltd 
(Trivellin et al. 2014, Beckers et al. 2015), and from HEK293 cells, as described previously (Trivellin et al. 2014). RNAseq analysis was performed by Zymo Research in pituitary tumor RNA samples from both X-LAG patients. Briefly, HiSeq $50 \times 2$ bp paired-end reads from RNAseq samples first went through quality control steps by trimming adaptors and low quality bases $(q$ score $<20)$ and were then analyzed using the Cufflinks software (v2.2.0) for transcript assembly and differential expression. Default parameters were used unless otherwise specified. BAM files were visualized with IGV v.2.3.61.

To precisely characterize the 5'-untranslated region (UTR) of GPR101, the SMARTer Rapid Amplification of cDNA Ends (RACE) cDNA Amplification Kit (Clontech Laboratories) was employed, following the manufacturer's instructions. The following reverse gene-specific primer (GSP) located within the CDS of GPR101 was used for 5'-RACE: 5'-CCCCAGCCGTAGAGTGGAGGAGTGC-3'. Samples that were analyzed by 5 '-RACE include matched RNA samples isolated from lymphocytes and the pituitary tumor of an X-LAG patient, and RNA isolated from HEK293 cells. The identity of the 5'-RACE products was confirmed by sequencing using the following primers: Isoform 1F 5'-GGGCCATGGGAAAAAGATGTA GAGA-3', Isoform 2F 5'-CGCTGCCTCTCAGAGCTT-3', Isoform 3F 5'-TTTTGCCTTTCTCCCCAGCT-3', Isoform $4 \mathrm{~F} \quad$ 5'-CCAACGAGAGAGTGCACACA-3', Isoforms 1-4commonR 5'-GCACGGTTGAGCGGATGAT-3'. The identity of the entire 3UTR of GPR101 determined by RNAseq was confirmed by using the following primer pairs: 3UTR_1F 5'-GGTGGGACTGAAGGCAAGAT-3' and 3UTR_1R 5'-ATTCTGCCTTCCTTGCTCCC-3'， 3UTR_2F 5'AGAAGTGGCCTAAGTCCTACAA-3' and 3UTR_2R 5'-GGGAATCTCAGTGCCCTTGA-3', 3'UTR_3F 5'-CCCCT TCAAAGTGTATCAGAGA-3' and 3'UTR_3R 5'-CTGGCAC TTTTCCTTACCTGT-3', 3UTR_4F 5'-TGGAAGCAGGGAA
GGAGAGA-3' and 3'UTR_4R 5'AGTGACCTGGCAATC TGTGG-3', 3UTR_5F 5'-GTCAAATGCCCAGCTTGCTT-3' and 3'UTR_5R 5'-ACCGTAGCTGTGACCCCATA-3', 3'UTR_6F 5'-CCCCTTTGGTTGGTTGTCTG-3' and 3UTR_6R 5'-TCAATTTCTCCGCATTTCCCA-3'.

There are some intrinsic limitations in the techniques employed: RNAseq experiments are limited by the number of reads covering specific regions in each sample making thus difficult to exactly determine the boundaries of transcripts. RACE products can be incomplete due to the premature termination of first-strand cDNA synthesis caused by pausing of the reverse transcriptase enzyme, especially for long RNAs (SMARTer RACE 5'/3' Kit User Manual, Clontech). To describe the beginning of each alternative 5UTR exon 1 and the end of the 3 UTR we decided to adopt a conservative approach and to report in the 'Results' section genomic coordinates that were verified by Sanger sequencing. Based on the location of the primers used for sequencing it is, therefore, possible that actual transcripts may vary by a few base pairs.

\section{mRNA expression analysis}

The expression levels of human GPR101 were measured by RT-quantitative PCR (qPCR) in a Human Normal cDNA Array containing 48 samples covering all major human normal tissues (HMRT103, Origene, Rockville, MD, USA). The TaqMan system using a ready-made probe-primer kit supplied from Applied Biosystems was used (Assay ID: Hs00369662_s1). This assay is located within GPR101 CDS and can detect all four isoforms. To determine the expression levels of each individual transcript, isoformspecific TaqMan assays were designed using the Primers Express v3.0 software (Applied Biosystems) (Table 1).

Total RNA was reverse transcribed to cDNA using the Superscript III Kit (Applied Biosystems) according to the

Table 1 TaqMan assays used to specifically detect the four different GPR101 isoforms.

\begin{tabular}{|c|c|}
\hline TaqMan assay & Primer/probe \\
\hline Isoform 1 & $\begin{array}{l}\text { Forward } \\
\text { Reverse } \\
\text { Probe }\end{array}$ \\
\hline Isoform 2 & $\begin{array}{l}\text { Forward } \\
\text { Reverse } \\
\text { Probe }\end{array}$ \\
\hline Isoform 3 & $\begin{array}{l}\text { Forward } \\
\text { Reverse } \\
\text { Probe }\end{array}$ \\
\hline Isoform 4 & $\begin{array}{l}\text { Forward } \\
\text { Reverse } \\
\text { Probe }\end{array}$ \\
\hline
\end{tabular}

Sequence (5'-3')
AAGAATCAGGAAACGGTGAGACA
CTGTTTACGACCGTCAGTTTCTGT
CGAGAGACACTGGAGAAG
TGCCTCTCAGAGCTTGCAG
GCACAGACCGCACTCAGT
CAGCAGCAACAGCATAGGC
CGGCAAAATAAAGTCCACAGGG
GAGACAGGTTGCAGGCTCAG
ACCAAGCATAGGCACTGAGT
CAGAGACACAGCGAACGACA
GAGACAGGTTGCAGGCTCAG
GAACAGCATAGGCACTGAGTG

\begin{tabular}{c}
\hline Assay length $(\mathrm{bp})$ \\
\hline 112 \\
227 \\
141 \\
101
\end{tabular}


manufacturer's protocols. The following samples were analyzed: pituitary tumors and lymphocytes of both X-LAG patients, lymphocytes of the unaffected parents of one X-LAG patient, two GH-secreting tumors without Xq26.3 microduplication, four normal pituitary glands, and HEK293 cells.

Human ACTB (Beta Actin, Applied Biosystems, 4326315E) was used as endogenous control in all reactions. TaqMan assays were performed according to manufacturer's protocol (Applied Biosystems) and analyzed using the $\Delta \Delta \mathrm{Ct}$ method, as described previously (Trivellin et al. 2014).

Total RNA was extracted from individual rat anterior pituitary glands using an RNeasy Plus Mini Kit (Qiagen) and reverse transcribed with a Transcriptor First Stand cDNA Synthesis Kit (Roche Applied Science). The expression levels of rat Gpr101 were measured by qPCR using the TaqMan system (Assay ID: Rn02084654_s1, Applied Biosystems) and normalized on Gapdh expression, as described previously (Bjelobaba et al. 2015).

Total RNA extracted from WT EK zebrafish embryos at different developmental stages was a generous gift of Dr Sadie Bergeron (National Institutes of Health $(\mathrm{NIH})$ ). One mcirogram of RNA was treated with DNase I (Applied Biosystems) to remove genomic DNA contamination and then reverse transcribed to cDNA using the Superscript III Kit (Applied Biosystems) according to the manufacturer's protocols. The expression levels of zebrafish gpr101 were measured by RT-PCR using the following primers: forward: TTCGTGCTCAACTTGCTCCT, reverse: CGCAGACA CTAGGGCAGAAT. Beta-actin (bactin1) was used as endogenous control (McCurley \& Callard 2008).

\section{Zebrafish embryo production}

WT EK zebrafish were used for all embryo production. All embryos were staged according to Kimmel et al. (1995).

\section{Whole-mount in situ hybridization}

A 1438 bp genomic sequence corresponding to the entire zebrafish gpr101 gene (ENSDARG00000039218) was cloned into the pCS2 vector and a DIG-labeled RNA antisense probe was synthesized by in vitro transcription (DIG RNA Labeling Kit (SP6/T7), Sigma-Aldrich) following manufacturer's protocol. The gh1 antisense probe was a generous gift of Dr Alberto Rissone (NIH). Whole-mount in situ hybridization (WISH) was then performed in WT EK embryos collected at different stages of development as described previously (Thisse $\&$ Thisse 2008). Staining with sense probes for both gpr101 and gh1 showed no staining, as expected (data not shown). Double WISH/immunofluorescence was performed as described previously (Barresi et al. 2005). The following antibodies were used: rabbit polyclonal anti-PRL (dilution 1:500, generous gift of Dr Akiyoshi Takahashi, Kitasato University, Japan), mouse monoclonal antityrosine hydroxylase (dilution 1:400, MAB318, clone LNC1, EMD Millipore) and appropriate rabbit/mouse secondary Alexa Fluor 488 (dilution 1:400, Thermo Fisher Scientific). Stained embryos were cleared in glycerol and photographed.

\section{Tissue collection and immunostaining}

Normal human pituitary tissues were collected during autopsy. Changes in cell density in the fetal pituitary were studied according to gestational age and location in the lateral or medial portions of the gland. There were 18 fetal pituitary samples from gestational ages ranging from 12 to 38 weeks. Of these, seven pituitary samples could be analyzed completely in lateral and medial portions on both sides of the developing gland. Formalin-fixed paraffin-embedded samples of whole fetal pituitaries preserved at autopsy were sectioned using standard techniques. All paraffin-embedded slides were submitted to de-parafinization, rehydration and antigen retrieval for $30 \mathrm{~min}$ in citrate buffer solution (pH 6.0). All slides were incubated with $10 \%$ normal donkey serum (NDS) for $1 \mathrm{~h}$ for blocking of nonspecific binding, and afterward incubated with the following primary antibodies: rabbit anti-GPR101 (dilution 1:500; SAB4503289, Sigma-Aldrich), goat anti-GH (dilution 1:100, sc-10364; Santa Cruz Biotechnology) overnight at $4^{\circ} \mathrm{C}$. All slides were incubated for $1-2 \mathrm{~h}$ with the following secondary antibodies: donkey antirabbit 555 (A-31572, Applied Biosystems) and donkey antigoat 488 (A-11055, Applied Biosystems), both at 1:500 dilution. Prolong gold mounting media with DAPI (P36934, Applied Biosystems) was used to set the slides. As negative control, a specimen's section was incubated under identical conditions without primary antibody. Fluorescence was analyzed with a Leica AF6000 microscope (Leica) at a 63x magnification with fixed time of exposure for all samples. Subsequently, images were deconvoluted with the supplied Leica image processing software (Leica).

Male and female rat and female Rhesus monkey pituitaries were isolated and fixed in Bouin's solution for

Published by Bioscientifica Ltd 
A

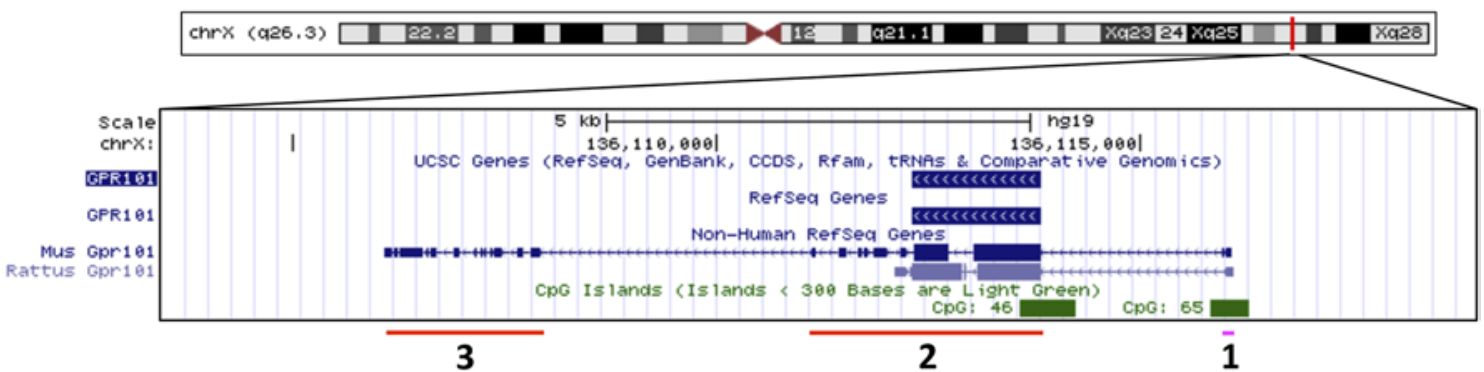

B

Color key for alignment score

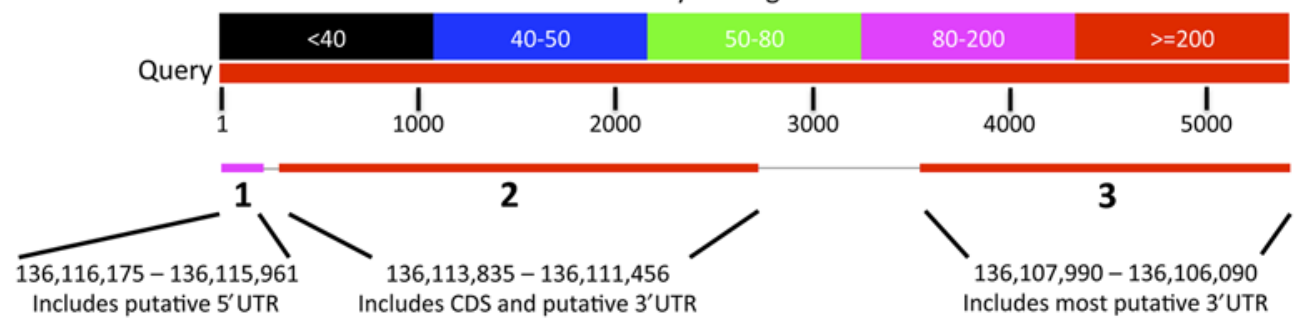

C

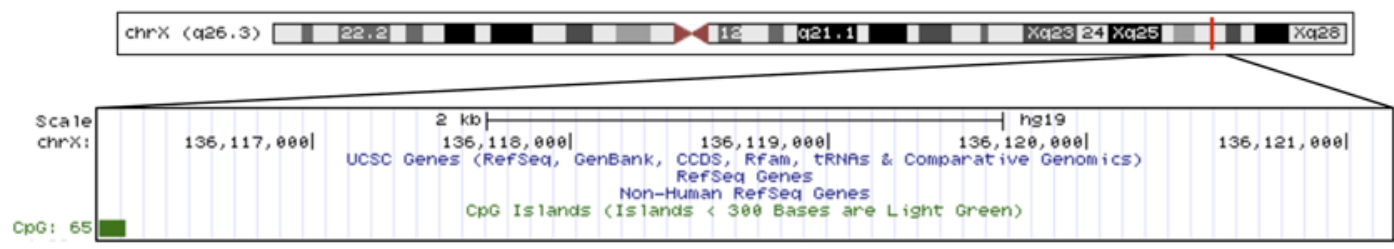

D

Color key for alignment score

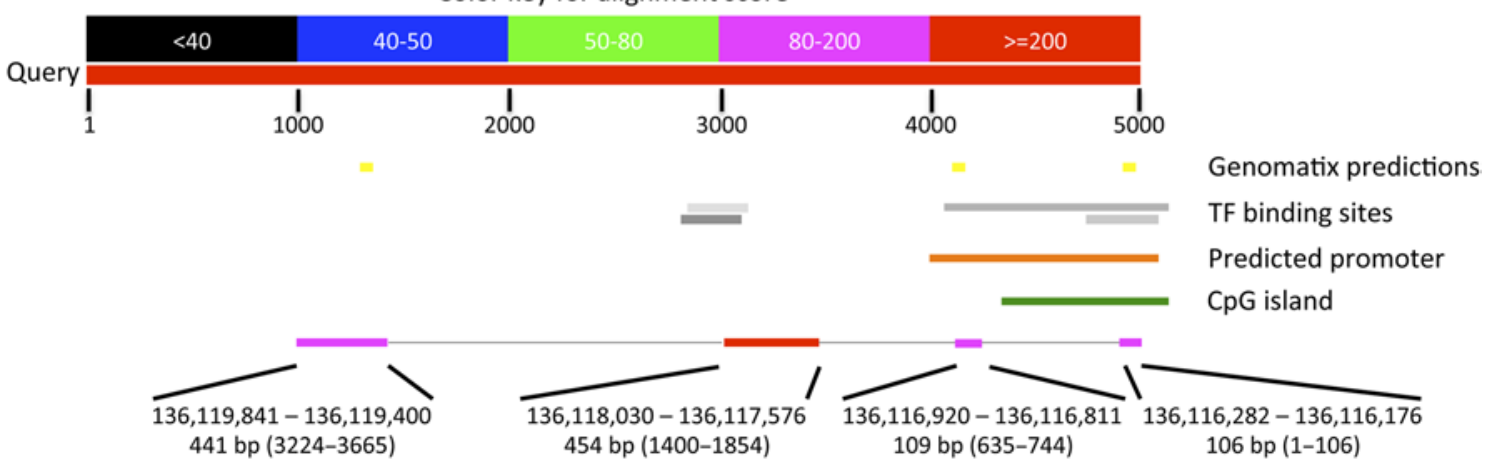

Figure 1

In silico analyses to predict human GPR101 promoter and untranslated regions. (A) This panel shows the human GPR101 aligned with mouse and rat Gpr101. The track displaying nonhuman RefSeq genes follows the display conventions for gene prediction tracks. The color shading indicates the level of review the RefSeq record has undergone: predicted (light), provisional (medium), reviewed (dark). The RNAs were aligned against the human genome using Blat. A track with the predicted CpG islands is also shown underneath. The number on the left of each island represents the CpG count. (B) The mouse Gpr101 mRNA was BLASTed to the human genome to determine the predicted UTRs of GPR101. The alignment predicted a $215 \mathrm{bp} 5$ UTR located about $2 \mathrm{~kb}$ upstream of the start codon and a splicing event in the $3^{\prime}$ UTR. (C and D) Analysis of the putative human GPR101 promoter region; $5 \mathrm{~kb}$ upstream of the predicted GPR101 TSS were aligned to the mouse genome (C). The alignment returned four regions which were further analyzed by MPromDb (orange bar), the UCSC genome browser track annotating TF binding sites assayed by ChIP-seq (gray bars) and Genomatix (yellow bars) (D). The identity of the predicted TFs is shown in Supplementary Fig. 1B and C. The location of the previously predicted CpG island by CpG Island Searcher (Trivellin et al. 2014) is also shown in panel D (green bar). A full colour version of this figure is available at http://dx.doi.org/10.1530/JME-16-0045. 
A

1

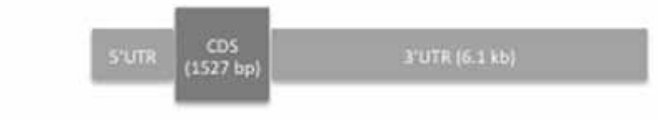

2

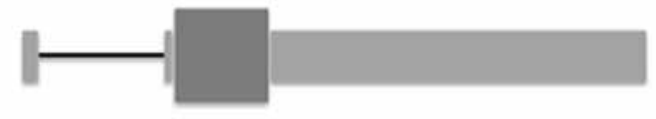

3

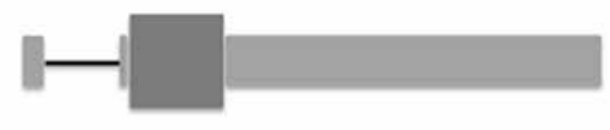

4

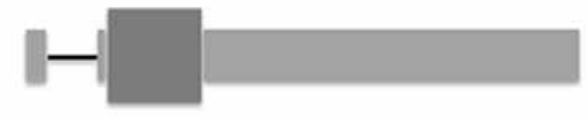

B

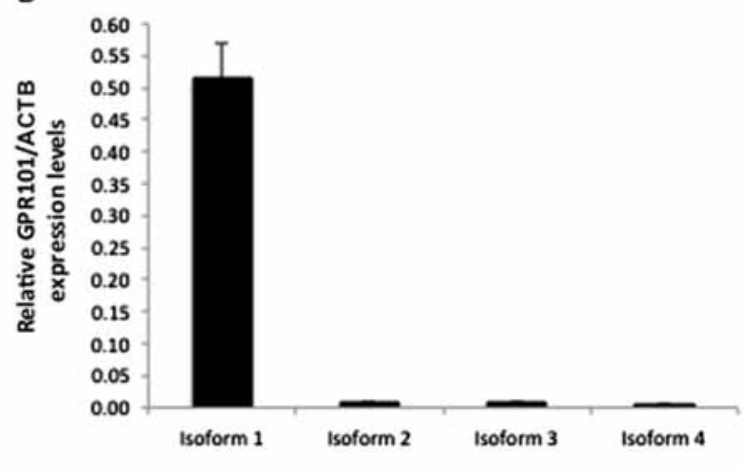

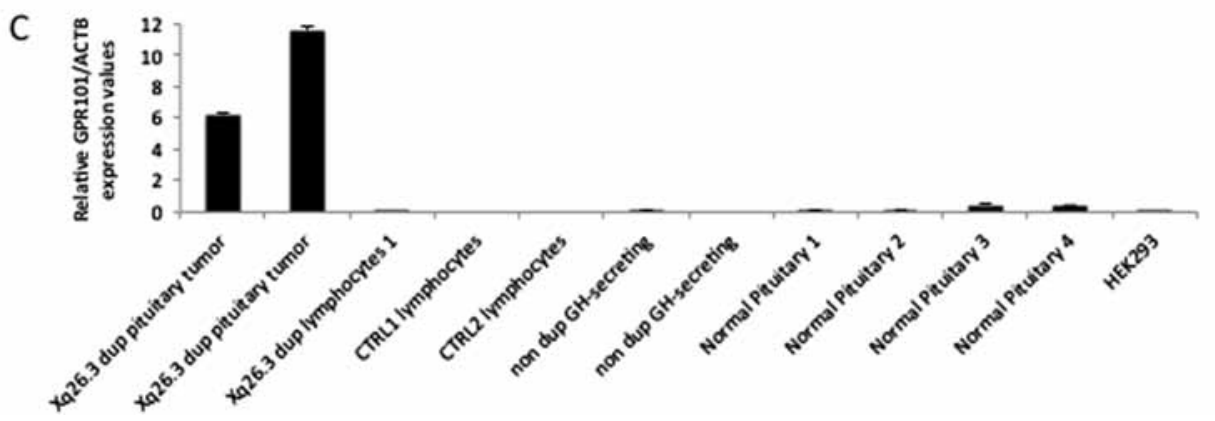

Figure 2

Structure and expression of GPR101 transcripts. (A) Four isoforms were observed in the analyzed samples by both 5'-RACE and RNAseq. All isoforms shared a common 3'UTR, while they differ for the 5'UTR. The coding sequence (CDS) is represented as a dark gray box, the UTRs as a smaller light gray box and the intron as a black line. (B) Relative expression of the four isoforms assessed by qPCR in a pituitary tumor harboring an Xq26.3 microduplication. Isoform 1 is expressed at much higher levels than the other isoforms. (C) Isoform 1 expression in different tissues. The relative expression of isoform 1 was determined by qPCR in the following samples: pituitary tumor and lymphocytes of an X-LAG patient, lymphocytes of her unaffected parents, normal pituitary, GH-secreting tumor without Xq26.3 microduplication, HEK293 cells. Isoform 1 expression was detected only in pituitary samples, showing higher expression in the tumor harboring a GPR101 duplication. All values are relative and normalized on ACTB expression.

$48 \mathrm{~h}$. Tissue was then processed for paraffin embedding. Five-micrometer-thick coronal sections of the pituitary tissues were deparaffinized and the heat-induced antigen retrieval was done in a microwave for $5 \mathrm{~min}$ with $10 \mathrm{mM}$ citrate buffer (pH 6). The rabbit polyclonal anti-GPR101 (SAB4503289, Sigma-Aldrich) was applied in $2 \mu \mathrm{g} / \mathrm{mL}$ dilution overnight at $4^{\circ} \mathrm{C}$. Immunofluorescence staining was performed using a Tyramide signal amplification kit (T20922, Invitrogen) with horseradish peroxidasegoat antirabbit IgG and Alexa fluor 488 tyramide, according to the manufacturer's instructions. Tissue was further processed for the second staining with another rabbit polyclonal antibody; to avoid falsepositive overlapping of signals, microwave treatment was used (Toth \& Mezey 2007) as described previously (Li et al. 2011). Rabbit antirat PRL, LH $\beta$, ACTH, TSH $\beta$, GH antisera were obtained from Dr AF Parlow (National Institute of Diabetes and Digestive and Kidney Diseases (NIDDK), National Hormone and Peptide Program,
Torrance, CA, USA) and used at a 1:400 dilution. For monkey tissue, rabbit anti-GH was obtained from DAKO (Dako North America, Inc, Carpinteria, CA, USA), 1:400 dilution. Donkey antirabbit Alexa fluor 647 was then applied at a 1:400 dilution for $2 \mathrm{~h}$. The sections were mounted in Mowiol and visualized under an inverted confocal microscope (Zeiss LSM 510). The same linear adjustments for brightness, contrast and color balance have been applied with Adobe Photoshop CS6 to each entire image.

\section{Statistical analysis}

For the mRNA expression experiments in rat pituitary tissue, a statistical analysis was performed with StatsDirect software (Addison-Wesley-Longman, Cambridge, UK). Data are presented as the mean \pm s.E.M. values from 6 to 37 pituitaries per group (Bjelobaba et al. 2015). Comparisons were calculated using a two-tailed Student's $t$-test for

Published by Bioscientifica Ltd. 

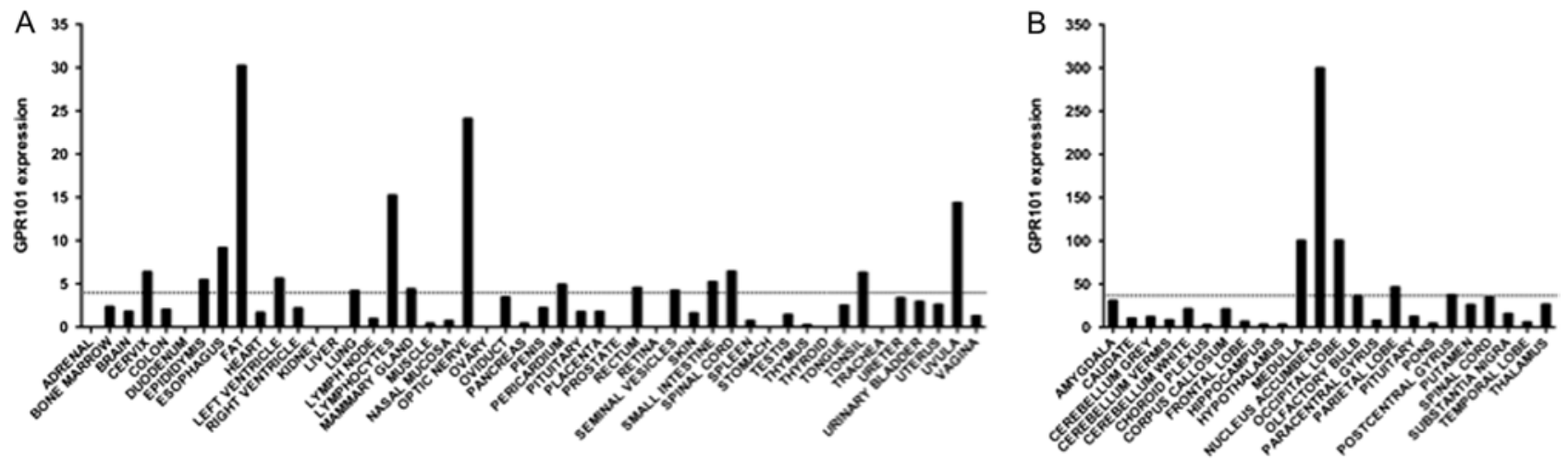

Figure 3

GPR101 mRNA expression in normal adult human tissues. (A) GPR101 expression in a panel of 48 different human tissues. (B) GPR101 expression in a panel of 24 human brain regions. The highest GPR101 expression levels were observed in the nucleus accumbens. All values are relative and were normalized on ACTB expression. The dotted lines represent the average expression in all tissues analyzed in each CDNA panel.

unpaired data. The data were considered to be significant when $P<0.05$.

\section{Results}

\section{In silico analysis to predict the promoter and untranslated regions of human GPR101}

For both mouse and rat Gpr101 orthologs, the UTRs have been characterized, whereas for the human GPR101 gene only the CDS, located on the reverse strand of chromosome $\mathrm{X}$ at 136,112,307-136,113,833 (hg19 assembly), has been characterized. Since the mouse Gpr101 RefSeq is reported as 'Validated', while the rat RefSeq status is 'Provisional', we decided to BLAST the mouse Gpr101 mRNA (NM_001033360.3) to the human genome. This alignment resulted in 74\% identity and $83 \%$ coverage. Three regions were aligned with the human genome, predicting the UTRs of GPR101: region 1 (215 bp from $136,115,961$ to $136,116,175)$, which includes the putative 5UTR; region 2 (2380 bp from 136,111,456 to $136,113,835)$, which includes the CDS and part of the putative 3UTR; and region 3 (1901 bp from 136,106,090 to $136,107,990)$, which includes most of the putative 3UTR (Fig. 1A). This analysis suggests that a splicing event occurs within the 3 UTR. The prediction of a long 3 UTR is also supported by the conservation of the DNA sequence in vertebrates (Supplementary Fig. 1A, see section on supplementary data given at the end of this article).

We then analyzed the putative human GPR101 promoter region by selecting $5 \mathrm{~kb}$ upstream of the predicted transcription start site (TSS) and aligning it to the mouse genome to look for conserved regions that are likely to harbor important transcriptional sites. This alignment resulted in 78\% identity and 21\% coverage. From this analysis, four regions were aligned with the human genome (Fig. 1B). We then further analyzed this $5 \mathrm{~kb}$ region by employing three different software programs: MPromDb (a mammalian promoter database), the UCSC genome browser track annotating TF binding sites assayed by ChIP-seq and Genomatix. MPromDb predicted a $1097 \mathrm{bp}$ promoter region located at $136,116,010-136,117,106$. The location of the TF binding sites predicted with the other two software packages are shown in Fig. 1B and Supplementary Fig. 1B and C. Taken together, these in silico analyses showed that the putative GPR101 promoter is likely located within $2 \mathrm{~kb}$ upstream of the predicted TSS, with the most important sites present within the first $1000 \mathrm{bp}$.

\section{In vitro characterization of GPR101 UTRs}

To confirm the in silico analysis, we characterized in vitro the UTRs of GPR101. Four GPR101 isoforms were identified in different human mRNA samples by the combination of 5'-RACE and RNAseq (Fig. 2A and Supplementary Fig. 2). The four isoforms are the result of alternative splicing at the 5'UTR, whereas they share a common 3'UTR that extends for $6.1 \mathrm{~kb}$ after the stop codon, until position $136,106,207$.

Isoform 1 consists of a single exon. The 5 UTR extends for $1300 \mathrm{bp}$ upstream of the start codon $(136,113,834$ $136,115,136)$. This isoform was observed in HEK293 cells and in all pituitary samples, albeit at different expression levels. In particular, the expression of this isoform in

Published by Bioscientifica Ltd 
HEK293 cells was at the limit of detection by qPCR, but we were nonetheless able to identify it in these cells by 5'-RACE PCR (Fig. 2C).

Isoform 2 consists of two exons and was observed by RNAseq and 5'-RACE PCR in the pituitary tumors of the two X-LAG patients and in HEK293 cells. The first exon is entirely noncoding and extends for $215 \mathrm{bp}(136,115,961$ $136,116,175)$, while the second exon consists of 92 noncoding bp upstream of the start codon $(136,113,834$ $136,113,925)$ and the $1527 \mathrm{bp}$ CDS. The two exons are separated by a 2035 bp long intron.

Isoforms 3 and 4 also consist of two exons and differ from isoform 2 at exon 1 . In isoform 3 , exon 1 extends for 302 bp $(136,115,139-136,115,440)$ and is separated from exon 2 by a 1213 bp long intron; in isoform 4, exon 1 extends for $295 \mathrm{bp}(136,114,730-136,115,024)$ and is separated from exon 2 by an 804 bp long intron. Both isoforms were found in the pituitary tumors of the two X-LAG patients; isoform 4 was also observed in HEK293
While we were able to detect isoforms 2, 3 and 4 by 5 -RACE PCR in different cells, their expression was detectable by qPCR only in the pituitary tumors of X-LAG patients, albeit at very low levels (Fig. 2B). Both intronic sequences of isoforms 2, 3 and 4 present classic donor and acceptor splice sites.

\section{GPR101 mRNA expression in adult human tissues}

To determine which tissues express GPR101 in humans, we performed qPCR in a panel of 48 normal adult tissues. GPR101 was generally expressed at low levels in all tissues analyzed (Fig. 3A). As Gpr101 was previously shown to be expressed predominantly in the brain in rodents, we analyzed GPR101 messenger levels in a panel of 24 different human brain regions. In adult humans, the highest GPR101 levels were observed in the nucleus accumbens. Other areas that showed relatively high expression were the medulla and the occipital lobe (Fig. 3B).
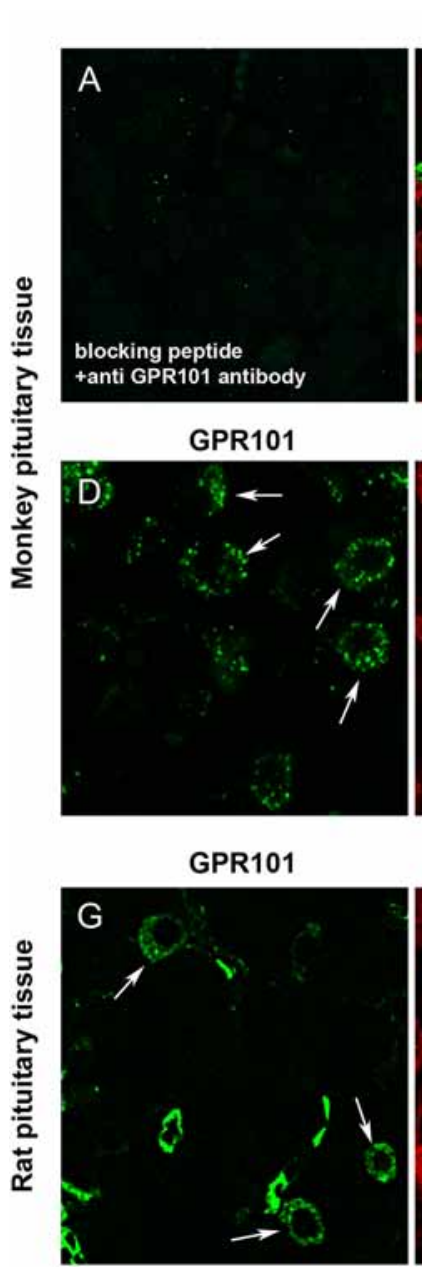
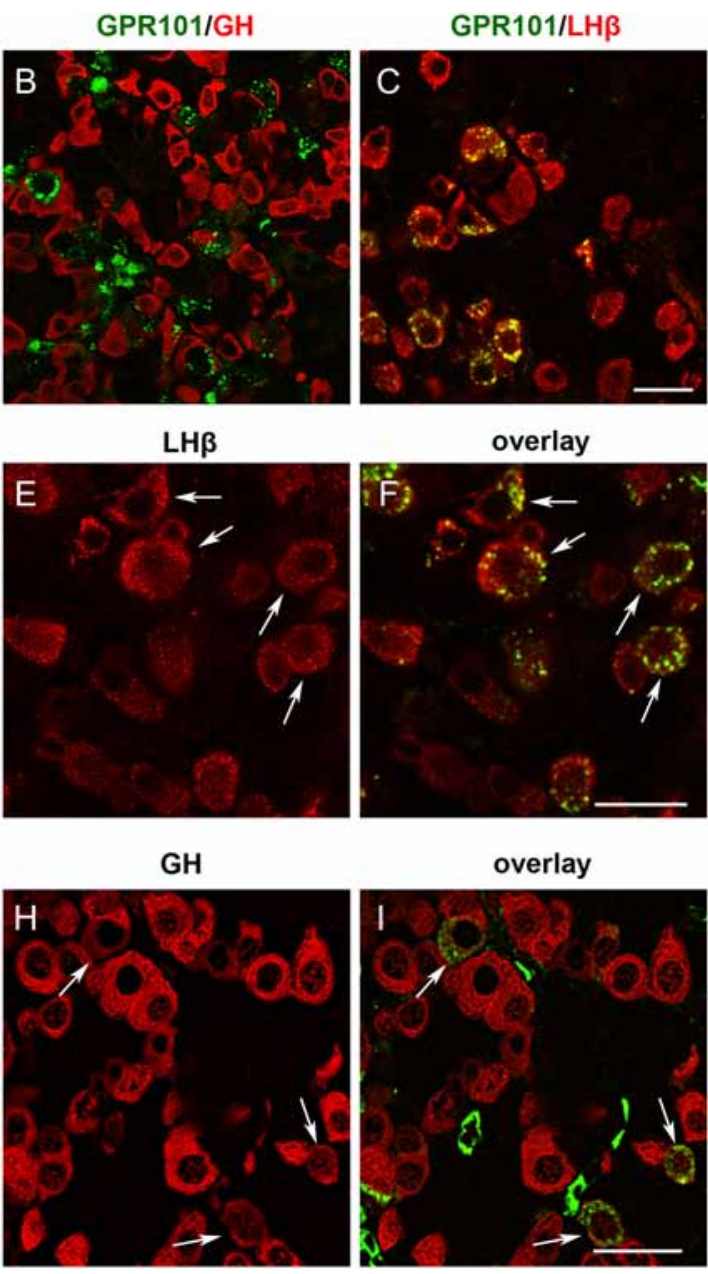

overlay

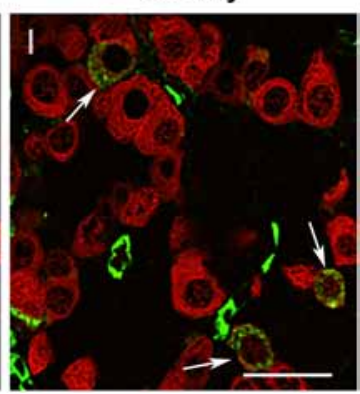

Figure 4

Labeling of GPR101 in the anterior pituitary gland of female rhesus monkey (A, B, C, D, E and F) and rat (G, $\mathrm{H}$ and I). (A) Preincubation of GPR101 antibody with blocking peptide, resulted in no staining. (B) Punctuate GPR101 (green) labeling could not be attributed to somatotrophs (red). (C) GPR101 (green) is expressed by LH $\beta$-positive cells (red). (D, E and F) High magnification images showing membranous and cytoplasmic distribution of GPR101 in monkey gonadotrophs, excluding nucleus. (G, H and I) GPR101 (green) is expressed by subpopulation of rat somatotrophs (red), as indicated by arrows. Scale bars: A, B, C and D: $20 \mu \mathrm{m}$; E: $10 \mu \mathrm{m}$. A full colour version of this figure is available at http:// dx.doi.org/10.1530/JME-16-0045. http://jme.endocrinology-journals.org DOI: 10.1530/JME-16-0045
C) 2016 Society for Endocrinology Printed in Great Britain
Published by Bioscientifica Ltd. 


\section{GPR101 expression in the adult anterior pituitary gland of other species}

We previously reported that GPR101 expression in the normal adult human pituitary is very low or absent (Trivellin et al. 2014). We confirm here our previous data by extending the analysis to further samples (Supplementary Fig. 3). To determine if this expression pattern is similar in other species, we assessed GPR101 expression in the adult pituitary of rhesus monkey and rat. In rhesus monkey pituitary tissue sections, GPR101 immunoreactivity was observed in gonadotroph cells, whereas all the other hormonesecreting cells stained negative (Fig. 4A, B, C, D, E and F). Immunohistochemical analysis in the rat pituitary showed a different expression pattern, with only GH-secreting cells expressing GPR101 (Fig. 4G, H, I and Supplementary Fig. 4).

\section{GPR101 expression during development}

In order to determine if the expression of GPR101

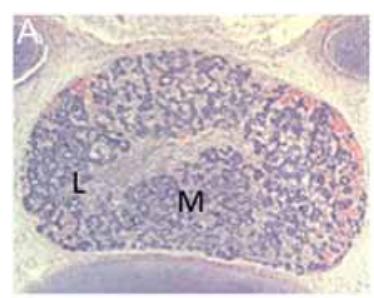

different stages of human growth, we performed GPR101 immunostaining in anterior pituitary tissues collected at autopsy during fetal development, early childhood and adolescence. GPR101-positive cells were absent or seen at very low levels in pituitary samples from before 19 weeks of gestation (Fig. 5A and B). From 19 to 25 weeks, about 20-30\% of cells were positive for GPR101 (Fig. 5C and D). Thereafter, positivity for GPR101 rose steadily from nearly $40 \%$ positive at 26 weeks to $>65 \%$ of cells positive at 38 weeks of gestation (Fig. 5E). However, studying medial and lateral locations separately, we found that GPR101 positivity in the medial portions of the pituitary remained markedly lower than that of the lateral portions throughout the fetal pituitary sample series.

In the pituitary tissue from a 2-year-old male child, very few if any GPR101-positive cells were seen (Fig. 5F). In contrast, we observed several GPR101-expressing cells in two male adolescent subjects aged 10 and 17 years old (Fig. 5G and H). As previously noted (Trivellin et al. 2014), the positive cells were not GH-positive. GPR101
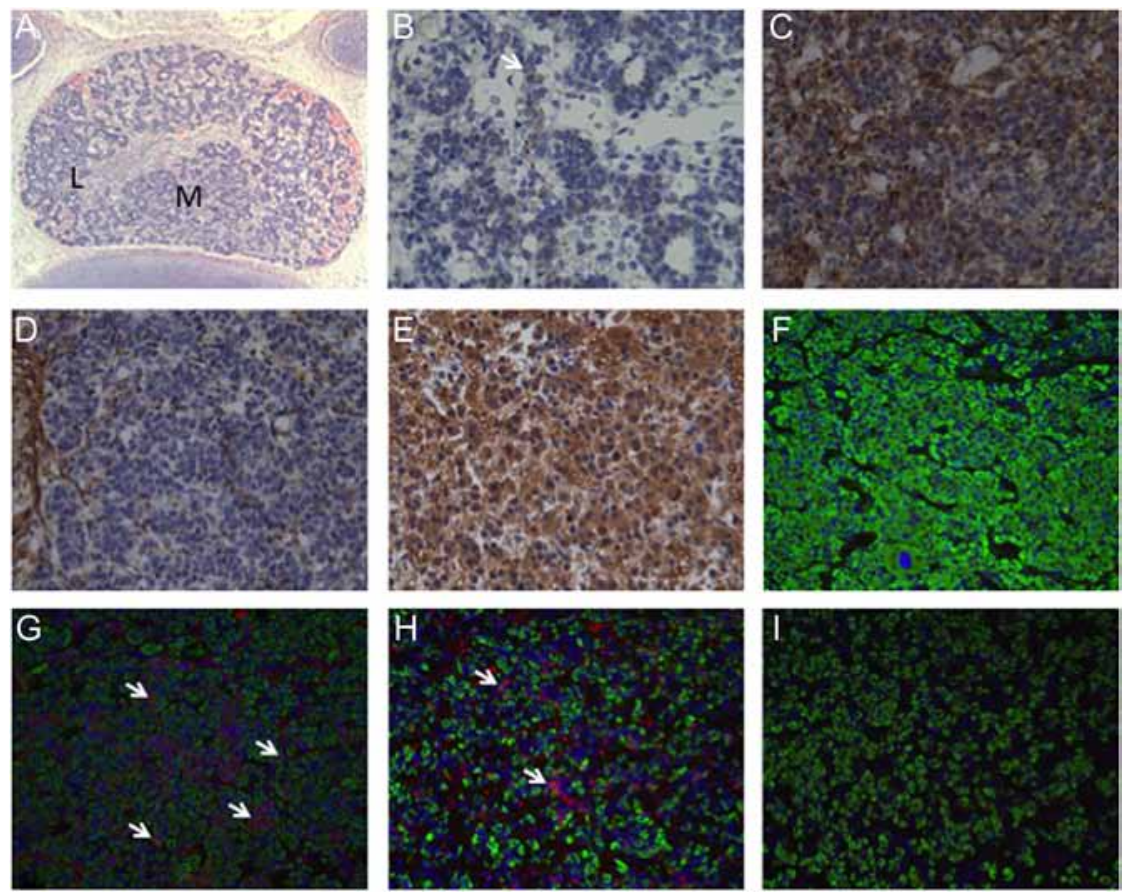

Figure 5

Human pituitary gland staining characteristics for GPR101. Panel A shows an H\&E stain transverse section of an entire pituitary gland from a 14-week-old fetus at low magnification (50x). The medial (M) and lateral (L) portions of the gland are highlighted. Panel B shows GPR101 staining (brown immunopositivity highlighted) in the lateral portion of a 14-week-old fetal pituitary at higher magnification (400x). Immunopositivity is localized to the cytoplasm and is negative in the nuclei. The different staining intensities for GPR101 at 24weeks in the lateral (Panel C) and medial (Panel D) portions of the fetal pituitary can be seen (400x). Some positivity for GPR101 in capillaries can also be seen. The increasing GPR101 staining intensity in the lateral portion of the fetal pituitary is illustrated in Panel E at 34weeks, where very high density is seen (400x). Panels F-I show GPR101 (red) and GH (green) immunofluorescence staining in the anterior pituitary during postnatal life. Almost absent GPR101 expression was seen in a 2-year-old female child (F) and in a 21-year-old male (I). Several GPR101-positive cells were present in two males aged $10(\mathrm{G})$ and $17(\mathrm{H})$ years old. Very little co-localization of GPR101 with GH was seen in panels G and H. Nuclei (blue) were counterstained with DAPI. A full colour version of this figure is available at http://dx.doi.org/10.1530/JME-16-0045.

http://jme.endocrinology-journals.org DOI: 10.1530/JME-16-0045
๑) 2016 Society for Endocrinology Printed in Great Britain
Published by Bioscientifica Ltd 

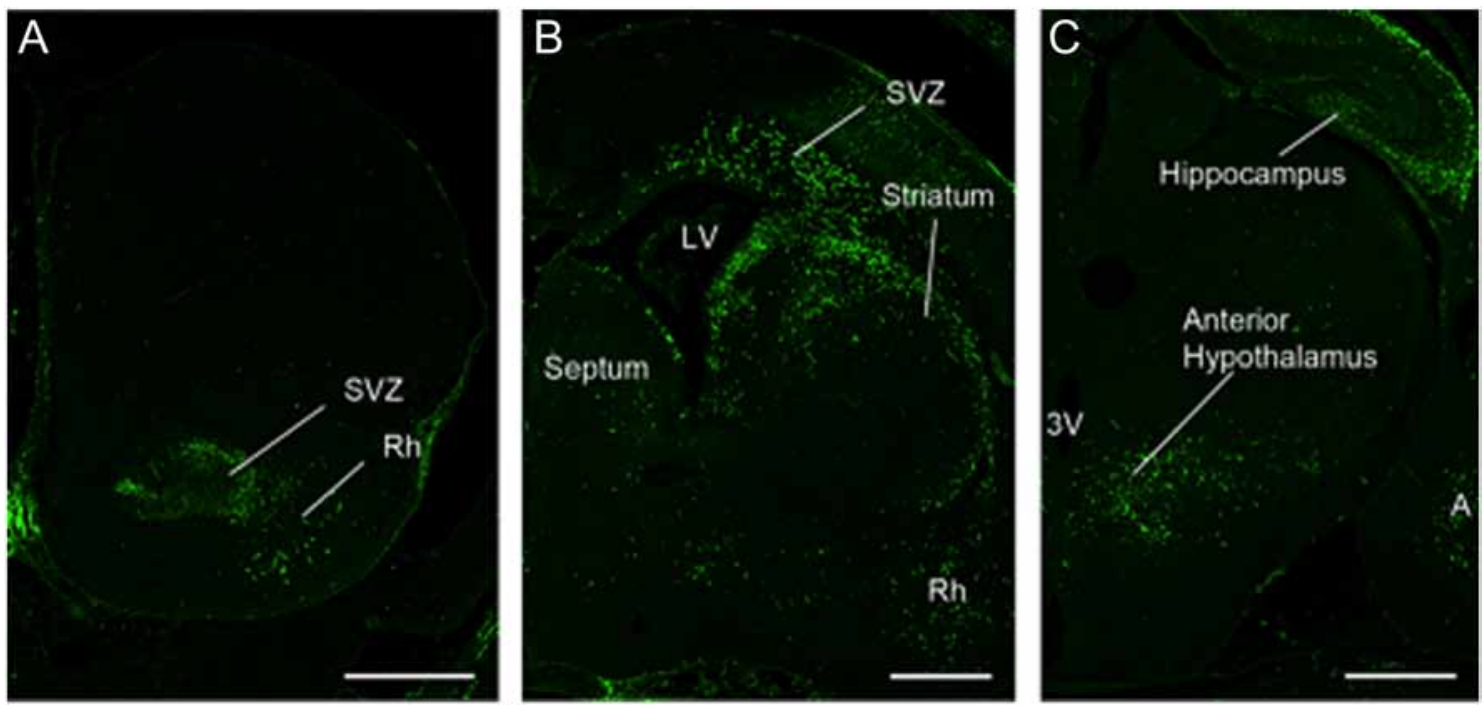

\section{Figure 6}

Immunolocalization of GPR101 in coronal sections of rat embryo forebrain. (A) GPR101 immunoreactive cells and processes were pronounced in accumbens subventricular zone (SVZ) and rhinencephalic differential field (Rh). (B) Strong GPR101 immunoreactivity was observed in striatal neuroepithelium and striatal subventricular zone (SVZ) around lateral ventricle (LV) and striatal differentiating field. GPR101-positive cells and cell processes were also observed in the septal area. (C) In more posterior sections, GPR101 immunoreactivity was observed throughout the hippocampus and lateral to third ventricle (3V), in the anterior hypothalamus. GPR101-positive cells and processes were also noticed in the amygdaloid differentiating field (A). Scale bar applies to all images: $0.5 \mathrm{~mm}$. A full colour version of this figure is available at http://dx.doi.org/10.1530/JME-16-0045.

expression was virtually absent in an adult 21-year-old male (Fig. 5I).

We also evaluated GPR101 expression during development in other species. GPR101 immunostaining in the forebrain of E20 female rat embryos was observed in several regions, including accumbens subventricular zone, rhinencephalon, striatal neuroepithelium, striatal subventricular zone, septum, hippocampus and anterior hypothalamus (Fig. 6). No sex differences were observed. We did not observe overlapping of GPR101 staining with vimentin (data not shown). In the prepubertal rat pituitary, Gpr101 mRNA expression showed sexual

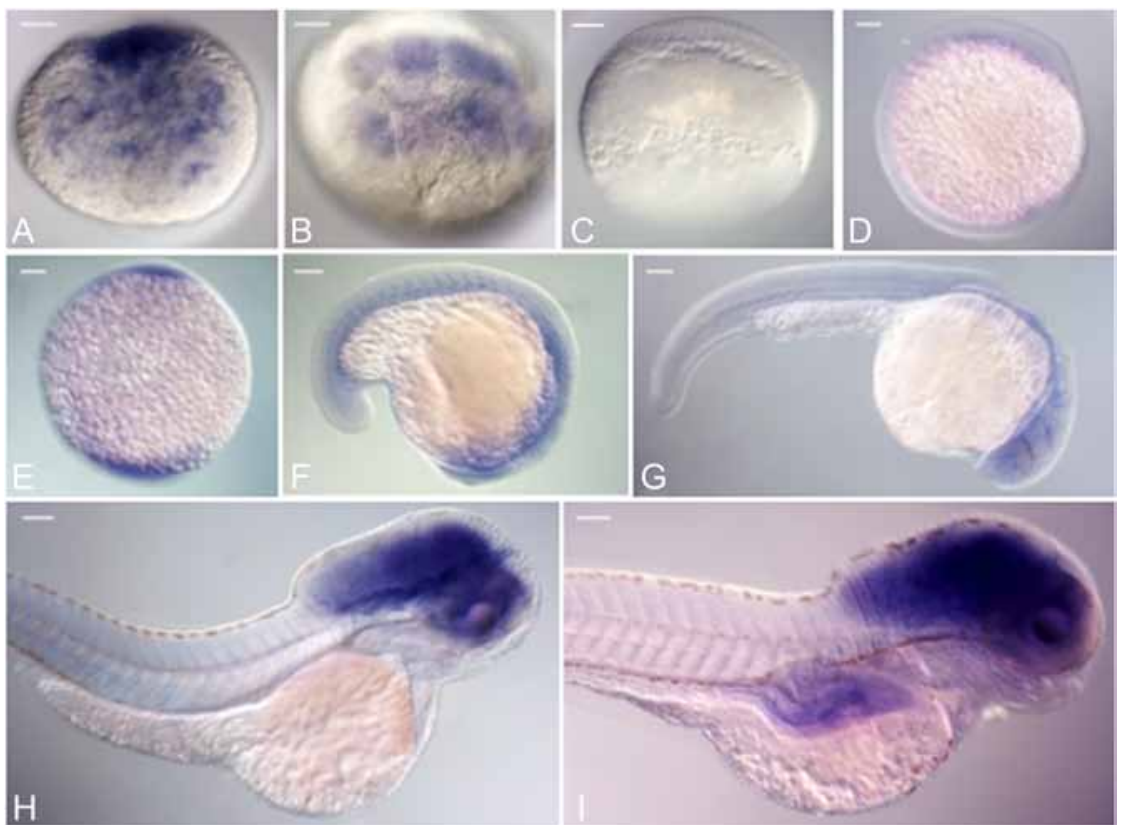

\section{Figure 7}

In situ staining for gpr101 during WT zebrafish development (lateral view). (A) 1-cell, (B) 8-cell, (C) $30 \%$ epiboly, (D) bud, (E) 3 somite, (F) 20 somite, (G) 24hpf, (H) 2dpf, (I) 5dpf. gpr101 mRNA (blue) is visible from 1-cell to 8-cell stage $(A, B)$, probably representing maternal transcripts, and then reappears during the bud stage (D), approximately $10 \mathrm{hpf}$. Starting at $2 \mathrm{dpf}(\mathrm{H})$ a strong and brain-specific staining is seen. Scale bar: $100 \mu \mathrm{m}$; dpf, days postfertilization; hpf, hours postfertilization. A full colour version of this figure is available at http://dx.doi.org/10.1530/ JME-16-0045. http://jme.endocrinology-journals.org DOI: 10.1530/JME-16-0045
C) 2016 Society for Endocrinology Printed in Great Britain
Published by Bioscientifica Ltd 
dimorphism during the first 3 weeks of life, with higher expression levels in females than males (Supplementary Fig. 5). An expression pattern similar to that observed in adult rat pituitary was also seen in prepubertal pituitary tissue by immunostaining (data not shown).

In the developing zebrafish embryo, we performed WISH using a gpr101-specific probe to determine at what stages and in which anatomical regions gpr101 is expressed. Transcripts of gpr101 showed a bimodal temporal expression pattern, with early, and likely maternal, messenger RNA levels waning during very early stages, then rising from the bud stage through $2 \mathrm{dpf}$, and remaining constant through the remainder of embryonic development (Fig. 7). This expression pattern
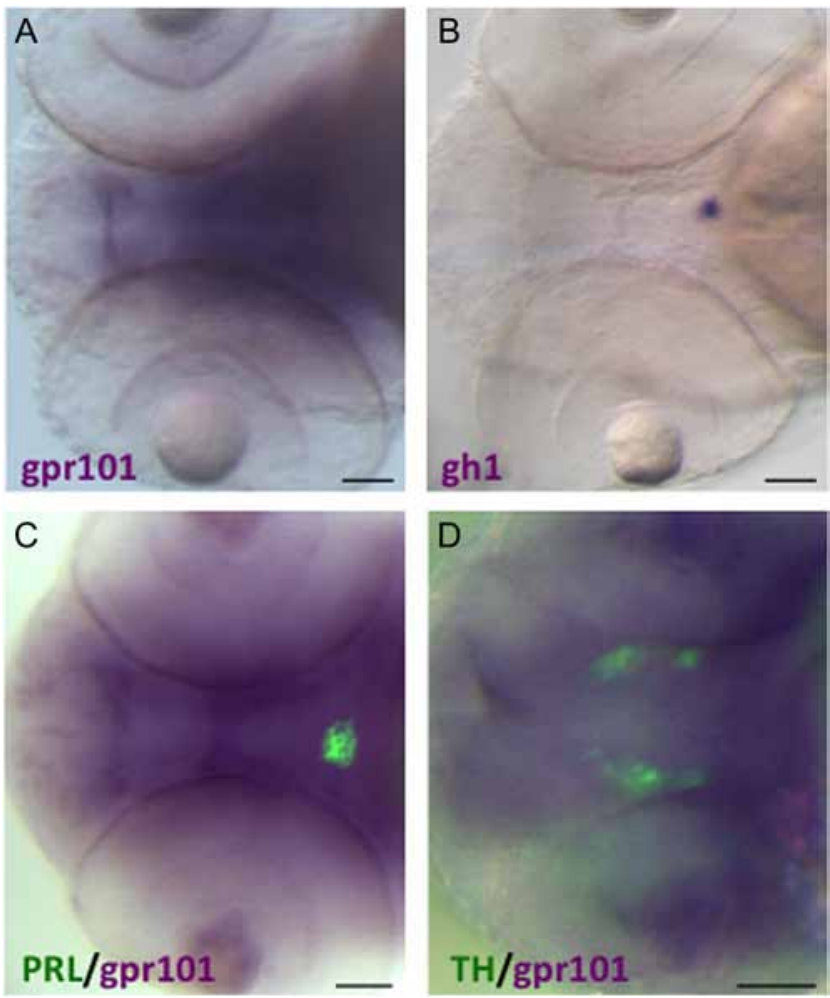

\section{Figure 8}

gpr101 expression in the brain of WT Zebrafish embryo. (A) and (B) WISH staining for gpr101 and gh1 (purple) in $2 \mathrm{dpf}$ distinct Zebrafish embryos (ventral view). gpr101 is strongly expressed in the whole brain region (A), while a pituitary-specific staining is visible with the gh1 probe (B). (C) and (D) Double WISH/immunofluorescence staining for gpr101 (purple) and a pituitary (PRL, green, panel C) and hypothalamic marker (tyrosine hydroxylase (TH) specific for diencephalic dopaminergic neurons, green, panel D) in a $2 \mathrm{dpf}$ Zebrafish embryo (ventral view). gpr101 staining overlaps with the location of pituitary and hypothalamic neurons. The qualitatively different aspect of gpr101 staining in panels $C$ and $D$ is due to the different focal planes selected for PRL and TH. Scale bar: $50 \mu \mathrm{m}$ applies to all panels. A full colour version of this figure is available at http://dx.doi.org/10.1530/JME-16-0045. was recapitulated when we measured gpr101 mRNA expression by RT-CR (Supplementary Fig. 6). Starting at $2 \mathrm{dpf}$, a strong and brain-specific staining was observed (Figs 7 and 8), overlapping with the location of pituitary and hypothalamus (Fig. 8).

\section{Discussion}

GPR101 is an orphan GPCR that is duplicated in patients with X-LAG. High GPR101 expression was observed in patients' pituitary tumor tissues, while low expression levels were seen in their blood and in normal pituitaries, suggesting that GPR101 is the likely cause of the disease (Trivellin et al. 2014). However, very little is known regarding GPR101 function, expression in different tissues and species, and transcript structure. We undertook this study in order to better understand GPR101 physiology and how its deregulation might cause X-LAG, and possibly other diseases (Tariq et al. 2013). Four GPR101 transcripts were identified. These transcripts are characterized by alternative splicing at the 5UTR due to different TSSs, while they share a common $6.1 \mathrm{~kb}$ long 3UTR.

Isoform 1 seems to be the predominant isoform in the human pituitary. The higher expression levels observed in the pituitary tumors harboring a duplication of GPR101 compared with normal pituitary tissue or tumors without the duplication suggests that this is the isoform that leads to the previously observed GPR101 overexpression (Trivellin et al. 2014). The other three isoforms are expressed at much lower levels and were readily detectable by qPCR only in GPR101-duplicated pituitary tumors. These isoforms might, therefore, represent minor transcripts, but we cannot exclude that they are specifically expressed in other tissues, where they might have a biological function. Isoform 3 was identified by both techniques only in mRNA isolated from pituitary tumors harboring an Xq26.3 microduplication. While it is possible that this is a pituitary-specific transcript, we could not exclude that this transcript might be generated due to the rearranged surrounding genomic region. Interplay between the tridimensional organization of the genome and the regulation of alternative splicing has indeed been previously shown (Curado et al. 2015).

Different approaches support the real nature of the identified transcripts. First, the in vitro data matched well with the in silico predictions. The predicted 5'UTR matches exactly with exon 1 of isoform 2 . Moreover, we previously showed that GPR101 is predicted to harbor a $747 \mathrm{bp} \mathrm{CpG}$ island (chrX: 136,115,961-136,116,708)

Published by Bioscientifica Ltd 
(Trivellin et al. 2014). Since CpG islands normally lie in the promoter region and can extend into the 5UTR (Medvedeva et al. 2010), this result is in line with the location of the identified 5'UTR. In addition, the canonical splicing sites identified for all alternative intronic regions support their bona fide nature. While we did not observe a splicing event within the 3UTR as inferred by the in silico alignment, the end of the predicted 3 UTR supports the in vitro observation. Second, concordant results were observed between the two techniques used for the in vitro characterization, RACE-PCR and RNAseq. It would be interesting for future studies to investigate the function of the very long 3 UTR, which could harbor several binding sites for regulatory molecules, such as miRNAs or lncRNAs.

Our in silico analyses showed that the putative GPR101 promoter region likely extends at least until $1 \mathrm{~kb}$ upstream of isoform 2 TSS (Fig. 1D). Several features suggest that the GPR101 promoter is broad and TATA-less, including the identification of a putative core promoter element associated with TATA-less genes (XCPE1, Supplementary Fig. 1C), the high GC content, and the multiple TSSs (Carninci et al. 2006, Sandelin et al. 2007, Tokusumi et al. 2007). A couple of putative TATA boxes were identified but their location far away from the TSS (Supplementary Fig. 1C) argues against a role in transcription activation. A conserved TATA box is indeed usually located around 30 bp upstream of the TSS (Sandelin et al. 2007). Future in vitro studies are necessary to decipher the function of the promoter region as well as of possible enhancers, which could lead to abnormally high GPR101 expression levels when rearranged.

Our analysis of GPR101 expression in adult human tissues showed that GPR101 is generally expressed at very low levels, with the exception of the brain, where relatively high expression was seen in several regions. This brain-specific expression correlates well with the in silico identification of a CpG-enriched promoter since this type of promoter could be advantageous in this organ due to its property of fine-tuning gene transcription (Gustincich et al. 2006). We also previously showed that when GPR101 is overexpressed, even in the absence of a ligand, it exhibits high constitutive activity by strongly activating the cAMP pathway (Trivellin et al. 2014). It is, therefore, conceivable that GPR101 expression is normally turned down or completely switched-off in most tissues, in line with our observations.

Within the brain, the highest GPR101 expression levels were observed in the nucleus accumbens, a region that plays an important role as the reward center and where other GPCRs characterized by constitutive activity play vital roles (Meye et al. 2014). This finding suggests that GPR101 might be involved in the regulation of different behaviors, such as food seeking: indeed, about a quarter of X-LAG cases presented with increased eating/food seeking that accompanied their markedly increased statural overgrowth (Beckers et al. 2015). Further studies are thus needed to determine if a functional link between GPR101, behavior and physical growth exists.

The pituitary gland, by responding to hormonal signals from the hypothalamus, constitutes an anatomical and physiological link between the nervous and the endocrine systems to control several key body functions, such as somatic growth. In order to gain insight into GPR101's putative role in regulating growth, we decided to investigate its expression in the brain and pituitary of different species both during and after development. GPR101 expression in the adult anterior pituitary gland showed remarkable differences among species. While GPR101 is expressed at low levels in adult human pituitary, GPR101-positive cells were seen in both monkey and rat pituitary. However, the cell types expressing the receptor differ in those two species. These results suggest that GPR101 might have different functions in the pituitary of different species during development and in adult life. In particular, in normal adult human pituitary, the main physiological role of GPR101 might already be finished. This is supported by the fact that high GPR101 expression was observed in the human pituitary gland during fetal development, which suggests that the receptor might play an important physiological role in this organ as it proliferates and matures. In particular, the observation that GPR101 is mainly expressed in the lateral wings of the anterior lobe, where the vast majority of GH- and PRL-secreting cells reside (Al-Gahtany et al. 2003, Melmed et al. 2011), suggests that the receptor might be involved in regulating, directly or indirectly, the embryonic differentiation of mammosomatotroph cells. Even if no causative association can be demonstrated by these data, it is interesting to note that in humans this is the stage were the maximum rate of growth in length and weight is achieved (Bogin 1999). Furthermore, duplication of GPR101 in the setting of X-LAG syndrome is associated with a specific pituitary pathology related to somatotroph, mammosomatotroph and lactotroph cells (Trivellin et al. 2014). It will be of interest in future studies to further define the main sites of hormonal dysregulation due to
() 2016 Society for Endocrinology Printed in Great Britain
Published by Bioscientifica Ltd 
GPR101 duplication in X-LAG syndrome, as we recently showed likely hypothalamic GHRH hypersecretion can accompany the pituitary pathology in this condition (Daly et al. 2016a).

We also investigated GPR101 expression in the pituitary during postnatal development. We observed very few GPR101-positive cells in normal pituitary tissue from a 2-year-old subject. This result seems to be in line with the observation that postnatal growth rates start to rapidly decelerate during infancy/early childhood (Bogin 1999, Stevens et al. 2013). We hypothesized that GPR101 might also play a role during the so-called 'adolescent growth spurt', a rapid and intense postpubertal increase in the rate of growth in height and weight that, among all primate species, is observed only in humans (Bogin 1999, Lui et al. 2015). This growth stage can account for up to $15-20 \%$ of adult final height (Cousminer et al. 2013) and, even if there is considerable variation between individuals and populations, begins on average at 10 years for girls and 12 for boys, and is clearly over by 18-19years for girls and 20-22years for boys (Bogin 1999, Lui et al. 2015). To test this hypothesis, we assessed GPR101 expression in pituitary tissues from adolescent individuals, to see if it might increase. We observed many GPR101-expressing cells in two male subjects aged 10 and 17 years old, whereas practically absent expression was seen in a 21-year-old male. These results indicate that GPR101 expression appears to increase during the adolescent growth spurt and then wanes once this growth phase terminates, thus supporting our hypothesis. However, we cannot exclude that GPR101 might also act at a higher (e.g. hypothalamic) level as suggested recently (Daly et al. 2016a). Altogether these results suggest that the expression of GPR101 in the human pituitary is tightly timed during pre- and postnatal development and lead us to speculate that the continuously high expression levels observed in the tumor tissues of patients with X-LAG compromise this fine regulation leading to uncontrolled growth.

GPR101 expression during pre- and postnatal development was also assessed in rat and zebrafish brain and pituitary. GPR101 was found to be expressed in several regions of the fetal rat brain, including the hypothalamus, but excluding the pituitary. These results are in agreement with what was previously observed in the adult rat brain (Bates et al. 2006). The absence of GPR101 expression in fetal rat pituitary could be explained by the fact that growth in rodents shows a very different course than in humans, with maximum growth rate occurring in the early postnatal period
(Stevens et al. 2013). Accordingly, GPR101 expression in the pituitary was detected only after birth, even if its expression levels were generally quite low. We observed sexual dimorphism with the highest relative GPR101 levels observed in 2-week-old females. Interestingly, at this point of female development, the highest mRNA levels were also observed for Gnrhr, Lhb, Fshb, and gonadotroph/thyrotroph-specific $\alpha$ subunit $(\mathrm{Cga})$ (Bjelobaba et al. 2015). These results lead us to speculate that in rodents, in contrast to humans, GPR101's putative role in growth is largely absent after birth. Moreover, GPR101-related effects seem to be exerted both in the pituitary and in higher brain regions (Bates et al. 2006, Nilaweera et al. 2007, 2008, Regard et al. 2008, Trivellin et al. 2014). In the zebrafish embryo, strong gpr101 expression was detected in the brain area. It will be important for future studies to generate brain regionspecific knockout animals in order to investigate the role(s) played by GPR101 during development.

In conclusion, this study shows that different human GPR101 transcripts exist and that the brain is the major site of GPR101 expression across different species, suggesting a conservation of function for this GPCR. On the other hand, we also observed species-specific expression patterns, especially concerning the pituitary, with GPR101 expression being regulated in a cell type- and developmental stage-specific manner in different species. Altogether, these findings indicate that (i) GPR101 plays an important role in both brain and pituitary development and (ii) the differences we observed might reflect the very different growth, development and maturation patterns among species.

\section{Supplementary data}

This is linked to the online version of the paper at http://dx.doi.org/10.1530/ JME-16-0045.

Declaration of interest

The authors declare that there is no conflict of interest that could be perceived as prejudicing the impartiality of the research reported.

\section{Funding}

This work was supported by the research project Z01-HD008920 (Principal Investigator: Dr Constantine A Stratakis) of the Intramural Research Program of the Eunice Kennedy Shriver National Institute of Child Health and Human Development (NICHD), National Institutes of Health (NIH), Bethesda, MD, USA. It was also supported, in part, by the Fonds d'Investissement pour la Recherche Scientifique (FIRS) of the Centre Hospitalier Universitaire de Liège and by NIH RO3HD078645 and National Science Foundation (NSF) IOS1052288 grants.

Published by Bioscientifica Ltd. 


\section{Acknowledgements}

The authors would like to thank Dr Alberto Rissone (National Institutes of Health (NIH), Bethesda, MD, USA) for providing the gh1 in situ probe; Dr Sadie Bergeron ( $\mathrm{NIH}$, Bethesda, MD, USA) for providing total RNA isolated from zebrafish embryos at different developmental stages; Dr Erica Bresciani (NIH, Bethesda, MD, USA) for technical help with the double WISH/immunofluorescence protocol; $\mathrm{Dr}$ Akiyoshi Takahashi (Kitasato University, Japan) for providing the anti-PRL antibody; $\mathrm{Dr}$ Laura C Hernández-Ramírez (NIH, Bethesda, MD, USA) for technical help, Stephanie Maquet, Lionel Masset and Dr Chantal Humblet of the GIGA Histology and Immunohistology Facility, University of Liège, Belgium for their excellent technical assistance.

\section{References}

Al-Gahtany M, Horvath E \& Kovacs K 2003 Pituitary hyperplasia. Hormones 2 149-158. (doi:10.14310/horm.2002.1149)

Barresi MJ, Hutson LD, Chien CB \& Karlstrom RO 2005 Hedgehog regulated Slit expression determines commissure and glial cell position in the zebrafish forebrain. Development 132 3643-3656. (doi:10.1242/dev.01929)

Bates B, Zhang L, Nawoschik S, Kodangattil S, Tseng E, Kopsco D, Kramer A, Shan Q, Taylor N, Johnson J, et al. 2006 Characterization of Gpr101 expression and G-protein coupling selectivity. Brain Research 1087 1-14. (doi:10.1016/j.brainres.2006.02.123)

Beckers A, Lodish MB, Trivellin G, Rostomyan L, Lee M, Faucz FR, Yuan B, Choong CS, Caberg JH, Verrua E, et al. 2015 X-linked acrogigantism syndrome: clinical profile and therapeutic responses. Endocrine-Related Cancer 22 353-367. (doi:10.1530/ERC-15-0038)

Bjelobaba I, Janjic MM, Kucka M \& Stojilkovic SS 2015 Cell type-specific sexual dimorphism in rat pituitary gene expression during maturation. Biology of Reproduction 93 21. (doi:10.1095/biolreprod.115.129320)

Bogin B 1999 Evolutionary perspective on human growth. Annual Review of Anthropology 28 109-153. (doi:10.1146/annurev. anthro.28.1.109)

Carninci P, Sandelin A, Lenhard B, Katayama S, Shimokawa K, Ponjavic J, Semple CA, Taylor MS, Engstrom PG, Frith MC, et al. 2006 Genome-wide analysis of mammalian promoter architecture and evolution. Nature Genetics 38 626-635. (doi:10.1038/ng1789)

Cousminer DL, Berry DJ, Timpson NJ, Ang W, Thiering E, Byrne EM, Taal HR, Huikari V, Bradfield JP, Kerkhof M, et al. 2013 Genome-wide association and longitudinal analyses reveal genetic loci linking pubertal height growth, pubertal timing and childhood adiposity. Human Molecular Genetics 22 2735-2747. (doi:10.1093/hmg/ddt104)

Curado J, Iannone C, Tilgner H, Valcarcel J \& Guigo R 2015 Promoterlike epigenetic signatures in exons displaying cell type-specific splicing. Genome Biology 16 236. (doi:10.1186/s13059-015-0797-8)

Daly AF, Lysy PA, Desfilles C, Rostomyan L, Mohamed A, Caberg JH, Raverot V, Castermans E, Marbaix E, Maiter D, et al. 2016a GHRH excess and blockade in X-LAG syndrome. Endocrine-Related Cancer 23 161-170. (doi:10.1530/erc-15-0478)

Daly AF, Yuan B, Fina F, Caberg JH, Trivellin G, Rostomyan L, de Herder WW, Naves LA, Metzger D, Cuny T, et al. 2016b Somatic mosaicism underlies $\mathrm{X}$-linked acrogigantism syndrome in sporadic male subjects. Endocrine-Related Cancer 23 221-233. (doi:10.1530/ erc-16-0082)

Gustincich S, Sandelin A, Plessy C, Katayama S, Simone R, Lazarevic D, Hayashizaki Y \& Carninci P 2006 The complexity of the mammalian transcriptome. Journal of Physiology 575 321-332. (doi:10.1113/ jphysiol.2006.115568)

Iacovazzo D, Caswell R, Bunce B, Jose S, Yuan B, HernandezRamirez LC, Kapur S, Caimari F, Evanson J, Ferrau F, et al. 2016 Germline or somatic GPR101 duplication leads to X-linked acrogigantism: a clinico-pathological and genetic study.
Acta Neuropatholica Communications 4 56. (doi:10.1186/s40478-0160328-1)

Kakarala KK \& Jamil K 2014 Sequence-structure based phylogeny of GPCR Class A Rhodopsin receptors. Molecular Phylogenetics and Evolution 74 66-96. (doi:10.1016/j.ympev.2014.01.022)

Kimmel CB, Ballard WW, Kimmel SR, Ullmann B \& Schilling TF 1995 Stages of embryonic development of the zebrafish. Developmental Dynamics 203 253-310. (doi:10.1002/ aja.1002030302)

Lee DK, Nguyen T, Lynch KR, Cheng R, Vanti WB, Arkhitko O, Lewis T, Evans JF, George SR \& O'Dowd BF 2001 Discovery and mapping of ten novel G protein-coupled receptor genes. Gene 275 83-91. (doi:10.1016/S0378-1119(01)00651-5)

Li S, Bjelobaba I, Yan Z, Kucka M, Tomic M \& Stojilkovic SS 2011 Expression and roles of pannexins in ATP release in the pituitary gland. Endocrinology 152 2342-2352. (doi:10.1210/en.2010-1216)

Lui JC, Garrison P \& Baron J 2015 Regulation of body growth. Current Opinion in Pediatrics 26 502-510. (doi:10.1097/ MOP.0000000000000235)

McCurley AT \& Callard GV 2008 Characterization of housekeeping genes in zebrafish: male-female differences and effects of tissue type, developmental stage and chemical treatment. BMC Molecular Biology 9 102. (doi:10.1186/1471-2199-9-102)

Medvedeva YA, Fridman MV, Oparina NJ, Malko DB, Ermakova EO, Kulakovskiy IV, Heinzel A \& Makeev VJ 2010 Intergenic, gene terminal, and intragenic CpG islands in the human genome. BMC Genomics 11 48. (doi:10.1186/1471-2164-11-48)

Melmed S, Kleinberg D \& Ho KEN 2011 Pituitary physiology and diagnostic evaluation. In Williams Textbook of Endocrinology, edn Twelfth Ed, pp 175-228. Boston, MA, USA: Elsevier Inc.

Meye FJ, Ramakers GMJ \& Adan RaH 2014 The vital role of constitutive GPCR activity in the mesolimbic dopamine system. Translational Psychiatry 4 e361. (doi:10.1038/tp.2013.130)

Mukhopadhyay S, Wen X, Ratti N, Loktev A, Rangell L, Scales SJ \& Jackson PK 2013 The ciliary G-protein-coupled receptor Gpr161 negatively regulates the Sonic hedgehog pathway via cAMP signaling. Cell 152 210-223. (doi:10.1016/j. cell.2012.12.026)

Nilaweera KN, Ozanne D, Wilson D, Mercer JG, Morgan PJ \& Barrett P $2007 \mathrm{G}$ protein-coupled receptor $101 \mathrm{mRNA}$ expression in the mouse brain: altered expression in the posterior hypothalamus and amygdala by energetic challenges. Journal of Neuroendocrinology 19 34-45. (doi:10.1111/j.1365-2826.2006.01502.x)

Nilaweera KN, Wilson D, Bell L, Mercer JG, Morgan PJ \& Barrett P 2008 G protein-coupled receptor 101 mRNA expression in supraoptic and paraventricular nuclei in rat hypothalamus is altered by pregnancy and lactation. Brain Research 1193 76-83. (doi:10.1016/ j.brainres.2007.11.048)

Regard JB, Sato IT \& Coughlin SR 2008 Anatomical profiling of G protein-coupled receptor expression. Cell 135 561-571. (doi:10.1016/j.cell.2008.08.040)

Rodd C, Millette M, Iacovazzo D, Stiles CE, Barry S, Evanson J, Albrecht S, Caswell R, Bunce B, Jose S, et al. 2016 Somatic GPR101 duplication causing X-linked acrogigantism (XLAG)-diagnosis and management. Journal of Clinical Endocrinology \& Metabolism 101 1927-1930. (doi:10.1210/jc.2015-4366)

Sandelin A, Carninci P, Lenhard B, Ponjavic J, Hayashizaki Y \& Hume DA 2007 Mammalian RNA polymerase II core promoters: insights from genome-wide studies. Nature Reviews Genetics 8 424-436. (doi:10.1038/ $\operatorname{nrg} 2026)$

Stevens A, Hanson D, Whatmore A, Destenaves B, Chatelain P \& Clayton P 2013 Human growth is associated with distinct patterns of gene expression in evolutionarily conserved networks. BMC Genomics 14 547. (doi:10.1186/1471-2164-14-547)

Tariq M, Cast AE, Belmont JW \& Ware SM 2013 Identification of a novel cause of X-linked heterotaxy. In Proceedings of the American Society of http://jme.endocrinology-journals.org

DOI: 10.1530/JME-16-0045
๑ 2016 Society for Endocrinology Printed in Great Britain 
Human Genetics 2013. Boston 22-26 October 2013. Program 227, Abstract 130122783. Bethesda, MD, USA: American Society of Human Genetics. (available at: http://www.ashg.org/2013meeting/ abstracts/fulltext/search_page-04.shtml)

Thisse C \& Thisse B 2008 High-resolution in situ hybridization to whole-mount zebrafish embryos. Nature Protocols 3 59-69. (doi:10.1038/nprot.2007.514)

Tokusumi Y, Ma Y, Song X, Jacobson RH \& Takada S 2007 The new core promoter element XCPE1 (X Core Promoter Element 1) directs activator-, mediator- and TATA-binding protein-dependent but TFIID-independent RNA polymerase II transcription from TATA-less promoters. Molecular and Cellular Biology 27 1844-1858. (doi:10.1128/MCB.01363-06)

Toth ZE \& Mezey E 2007 Simultaneous visualization of multiple antigens with tyramide signal amplification using antibodies from the same species. Journal of Histochemistry \& Cytochemistry $\mathbf{5 5}$ 545-554. (doi:10.1369/jhc.6A7134.2007)

Trivellin G, Daly AF, Faucz FR, Yuan B, Rostomyan L, Larco DO, Schernthaner-Reiter MH, Szarek E, Leal LF, Caberg JH, et al. 2014 Gigantism and acromegaly due to Xq26 microduplications and GPR101 mutation. New England Journal of Medicine 371 2363-2374. (doi:10.1056/NEJMoa1408028)

Received in final form 23 May 2016

Accepted 9 June 2016

Accepted Preprint published online 9 June 2016
Published by Bioscientifica Ltd. 